\title{
Uma abordagem atual da utilização de antidepressivos no manejo da depressão pós-parto
}

Thiago Guimarães Da-Silva ${ }^{1}$

(D) https://orcid.org/0000-0002-8321-3647

Pedro Fonseca de Vasconcelos ${ }^{1}$

(D) https://orcid.org/0000-0003-4289-0753

Ivan Gilson Silva Moura ${ }^{1}$

(D) https://orcid.org/0000-0003-2203-7632

Faculdade de Saúde Santo Agostinho, Campus Vitória da Conquista, Vitória da Conquista, BA, Brasil.
Objetivo: caracterizar a utilização de antidepressivos no manejo da depressão pós-parto. Método: empregou-se uma revisão integrativa de literatura, das bases de dados PubMed e Biblioteca Virtual em Saúde, com aplicação de descritores, visando responder a pergunta norteadora do trabalho, entre os dias 25 de fevereiro e 10 de março de 2019. Com base nos critérios de inclusão e exclusão, foram selecionados 23 artigos que, posteriormente, foram submetidos à categorização. Resultados: a sertralina deve ser a droga de escolha para o tratamento farmacológico da depressão puerperal. Constatouse também, que a utilização profilática de antidepressivos em mulheres susceptíveis é contestável e pouco se sabe sobre os possíveis efeitos colaterais. Ademais, foi encontrado que não há consenso sobre a superioridade da terapia farmacológica em detrimento às psicoterapias. Conclusão: há evidencias que fundamentam o uso de sertralina, paroxetina, duloxetina, nortriptilina e imipramina para tratar mulheres com depressão pós-parto, sendo a amamentação sempre recomendada. Ressalta-se que emerge a necessidade de estudos com amostras representativas para validar ou restringir o uso de psicofármacos na profilaxia da depressão puerperal.

Descritores: Depressão Pós-Parto; Psicotrópicos; Agentes Antidepressivos; Sertralina.

\section{Como citar este artigo}

Da-Silva TG, Vasconcelos PF, Moura IGM. A current approach to the use of antidepressants in the management of postpartum depression. SMAD, Rev Eletrônica Saúde Mental Álcool Drog. 2021 jan.- mar.;17(1):101-108. doi: https://dx.doi.org/10.11606/issn.1806-6976.smad.2021.159781 


\title{
A current approach to the use of antidepressants in the management of postpartum depression
}

\begin{abstract}
Objective: this study aimed to characterize the use of antidepressants in the management of postpartum depression. Method: an integrative literature review of the PubMed and Virtual Health Library databases was used, with the application of descriptors, aiming to answer the guiding question of the work, between February $25^{\text {th }}$ and March $10^{\text {th }}, 2019$. Based on the inclusion and exclusion criteria, 23 articles were selected that were later submitted to categorization. Results: sertraline should be the drug of choice for the pharmacological treatment of puerperal depression. It was also found that the prophylactic use of antidepressants in susceptible women is controversial and little is known about the possible side effects. In addition, it was found that there is no consensus on the superiority of pharmacological therapy to the detriment of psychotherapies. Conclusion: there is evidence supporting the use of sertraline, paroxetine, duloxetine, nortriptyline and imipramine to treat women with postpartum depression, and breastfeeding is always recommended. It is worth noting that the need for studies with representative samples to validate or restrict the use of psychotropic drugs in the prophylaxis of puerperal depression emerges.
\end{abstract}

Descriptors: Postpartum Depression; Psychotropics; Antidepressant Agents; Sertraline.

\section{Enfoque actual de la utilización de antidepresivos en el manejo de la depresión postparto}

Objetivo: el presente estudio tuvo como objetivo caracterizar la utilización de antidepresivos en el manejo de la depresión posparto. Método: revisión integradora de literatura, de las bases de datos PubMed y Biblioteca Virtual de Salud, con aplicación de descriptores, para responder a la pregunta orientadora del trabajo, entre el 25 de febrero y el 10 de marzo de 2019. Con base en los criterios de inclusión y exclusión, se seleccionaron 23 artículos que posteriormente se sometieron a categorización. Resultados: la sertralina debe ser la droga elegida para el tratamiento farmacológico de la depresión puerperal. Además, se constató que la utilización profiláctica de antidepresivos en mujeres susceptibles es discutible y poco se sabe sobre los posibles efectos colaterales. Asimismo, se encontró que no hay consenso sobre la superioridad de la terapia farmacológica en detrimento de las psicoterapias. Conclusión: hay evidencias que fundamentan el uso de sertralina, paroxetina, duloxetina, nortriptilina e imipramina para tratar a mujeres con depresión posparto, siendo la lactancia siempre recomendada. Se destaca que surge la necesidad de realizar estudios con muestras representativas para validar o restringir el uso de psicofármacos en la profilaxis de la depresión puerperal.

Descriptores: Depresión Postparto; Psicotrópicos; Agentes Antidepresivos; Sertralina. 


\section{Introdução}

A depressão é a principal causa de incapacidade em todo o mundo(1). A desordem psiquiátrica em questão é mais frequente no período pós-parto do que em outras épocas da vida, afetando cerca de $10-15 \%$ das mães adultas e tem como principais consequências o maior risco de suicídio e infanticídio(2-4).

Essa desordem mental está associada com entraves na interação mãe-criança que ocorrem, pelo menos em parte, devido ao impacto da depressão na percepção materna negativa da expressão facial de seu bebê(5). Embora a abordagem dessa patologia inclua outras modalidades terapêuticas, os antidepressivos ainda são, em sua maioria, as drogas de escolha(1).

Os principais fatores de risco são de natureza social, geralmente decorrentes de vida estressante, estresse no cuidado da criança e ansiedade pré-natal. Os sintomas são iguais aos da depressão em outros momentos da vida, mas além de humor deprimido, irritabilidade, distúrbios do sono e mudança no apetite, as mães também se sentem culpadas por sua incapacidade de cuidar do novo bebê(2). Uma hipótese plausível para gênese da depressão pós-parto é a de que, no período puerperal, o declínio do estradiol e o aumento das enzimas monoamina oxidase resultem em piora no humor materno(6).

O tratamento farmacológico da depressão puerperal tem recebido muita atenção nos últimos anos, sendo que, na prática atual, cerca de $70 \%$ dos casos são tratados com antidepressivos ${ }^{(1,4)}$. Ademais, tem se mostrado relevante a discussão acerca desse tema, especialmente, devido aos possíveis efeitos teratogênicos e à toxicidade neonatal das drogas antidepressivas $^{(4)}$.

Portanto, frente à importância da temática em questão, o presente trabalho tem a seguinte questão norteadora: Como os antidepressivos tem sido utilizados no manejo da depressão pós-parto? Assim sendo, o presente artigo tem o objetivo de caracterizar a utilização de antidepressivos no manejo da depressão pós-parto, permitindo abordar a visão de diferentes autores acerca das drogas de escolha e seus efeitos adversos, bem como a utilização de terapias alternativas, como a psicoterapia.

\section{Método}

O presente trabalho realizou uma revisão integrativa de literatura para caracterizar as perspectivas atuais da utilização de antidepressivos no manejo da depressão pós-parto, seguindo o percurso apresentado na Figura 1, baseado em Souza, Silva e Carvalho(7).

A pesquisa recorreu às bases de dados PubMed e BVS (Centro Latino-Americano e do Caribe de Informação em Ciências da Saúde). Foram utilizados os seguintes Descritores em Ciências da Saúde e suas associações: Depressão Pós-Parto e Antidepressivos; com auxílio do operador booleano AND. A busca nas bases de dados ocorreu entre os dias 25 de fevereiro e 10 de março de 2019.

Executou-se a seleção dos artigos em duas etapas. Inicialmente fez-se a leitura dos resumos e, logo depois, procedeu-se a leitura completa dos artigos selecionados na primeira etapa, com base nos critérios de inclusão e exclusão. Os critérios de inclusão utilizados foram: a) estudos que identificaram a droga de escolha para manejo da depressão pós-parto; b) trabalhos que avaliaram a utilização profilática de antidepressivos em mulheres susceptíveis à depressão puerperal; c) publicações que avaliaram a segurança e/ou efeitos adversos de antidepressivos na vigência do aleitamento materno; d) artigos que compararam a eficácia de psicofármacos e psicoterapias no manejo da depressão pós-parto; e) período de publicação de 2009 a 2018; f) publicações em inglês, espanhol e português.

Como critérios de exclusão foram utilizados: a) data de publicação anterior a 2009; b) estudos em outro idioma além do inglês, espanhol ou português; c) artigos que não respondiam à pergunta norteadora da presente pesquisa. Após a aplicação desses critérios, foram selecionados 23 artigos pertinentes ao tema.

$\mathrm{O}$ artigos selecionados foram agrupados, de acordo com seus resultados, nos seguintes temas: Benefícios na utilização da sertralina para tratamento de depressão pós-parto; Eventos adversos e/ou segurança na terapia antidepressiva no aleitamento materno; Psicoterapia e psicofarmacologia no manejo da depressão pós-parto. Posteriormente, foram categorizados e discutidos nos seguintes tópicos: Considerações para utilização de antidepressivos; Droga de escolha e introdução da terapia farmacológica com base na gravidade da doença; Utilização profilática de antidepressivos em mulheres susceptíveis; Antidepressivos, aleitamento materno e efeitos adversos no lactente; Tratamento farmacológico vs. Psicológico. 


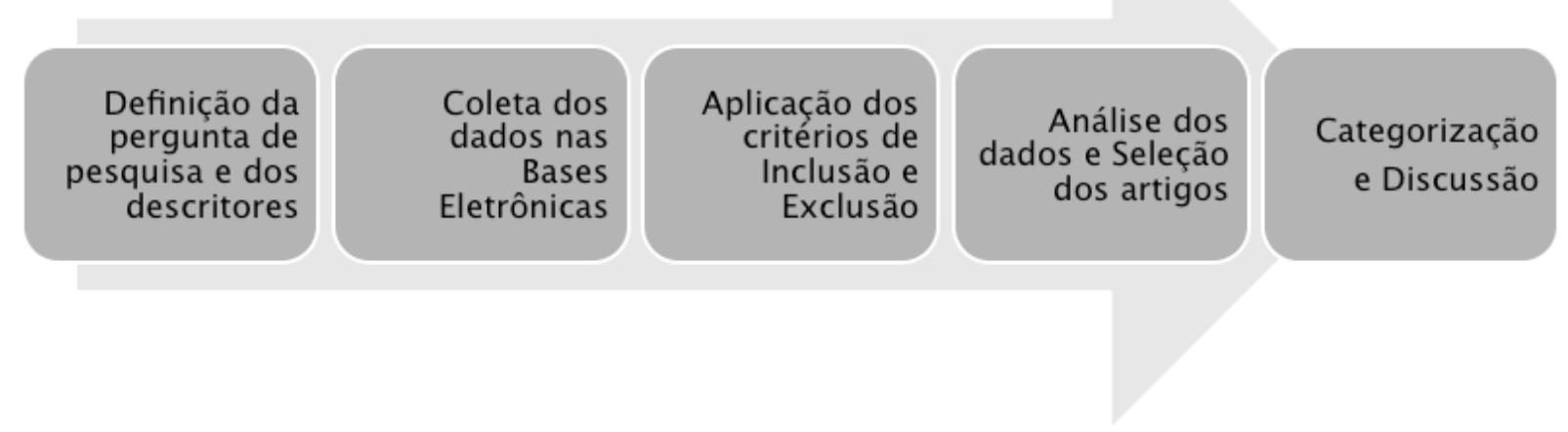

Figura 1 - Percurso metodológico da coleta e análise de dados na revisão integrativa para caracterizar as perspectivas atuais da utilização de antidepressivos no manejo da depressão pós-parto

\section{Resultados}

Os resultados encontrados na pesquisa demonstram que mulheres na vida reprodutiva são susceptíveis a interferências no mecanismo de ação de vários antidepressivos por efeitos do estrogênio, individualidades genéticas, alterações no volume de distribuição, efeitos hormonais nas enzimas hepáticas e maior concentração de proteínas que se ligam aos fármacos ${ }^{(3,8)}$. Deve-se ressaltar que a utilização de antidepressivos como terapia inicial é recomendada apenas na depressão pós-parto grave, sendo os demais casos tratados com psicoterapias ${ }^{(1,9-10)}$.

Diversos autores concordam que, quando se opta por iniciar a terapia farmacológica, a sertralina deve ser a droga de escolha para o tratamento da depressão pósparto. A Figura 2 expõe achados de estudos que apoiam a preferência por esta droga.

Quanto à utilização profilática de antidepressivos em mulheres susceptíveis, encontrou-se que a eficácia desses fármacos é contestável e pouco se sabe sobre os possíveis efeitos colaterais ${ }^{(11-12)}$. Contudo, se o médico decidir iniciar a terapia antidepressiva profilática, a sertralina é a droga indicada pela maioria das publicações ${ }^{(6,13-14)}$.

\begin{tabular}{|ll|}
\hline AUTORESIANO & RESULTADOS \\
\hline $\begin{array}{l}\text { Molenaar et al. } \\
(2018)\end{array}$ & $\begin{array}{l}\text { Baixa concentração no leite materno e no soro } \\
\text { das crianças }\end{array}$ \\
Kim et al. (2014) & $\begin{array}{l}\text { Resposta terapêutica eficaz em até } 67 \% \text { dos } \\
\text { casos }\end{array}$ \\
Kim et al. (2014) & Recorrência da doença de apenas 7\% \\
$\begin{array}{l}\text { Logsdon et al. } \\
(2009)\end{array}$ & Melhora da gratificação do papel materno \\
$\begin{array}{l}\text { Hantsoo et al. } \\
(2014)\end{array}$ & $\begin{array}{l}\text { Taxa de resposta significativamente maior que o } \\
\text { placebo (53\% vs. 21\%) }\end{array}$ \\
\hline
\end{tabular}

Figura 2 - Estudos que apresentam as evidências dos benefícios na utilização da sertralina para tratamento da depressão pós-parto
A amamentação fornece diversos benefícios à díade mãe-bebê e, por isso, deve ser recomendada, independente da medicação antidepressiva utilizada. Além de influenciar na aceitação da farmacoterapia, sua interrupção pode estar envolvida na gênese da depressão pós-parto(1,15-16). A Figura 3 expõe achados de estudos que avaliaram eventos adversos e/ou segurança das terapias antidepressivas no aleitamento materno.

\begin{tabular}{|c|c|}
\hline AUTORESIANO & RESULTADOS \\
\hline Di Scalea; Wisner (2009) & $\begin{array}{l}\text { Sertralina, paroxetina, nortriptilina e } \\
\text { imipramina associam-se a níveis séricos } \\
\text { indetectáveis no lactente e a nenhum } \\
\text { relato de efeito adverso em curto prazo. }\end{array}$ \\
\hline Andrade (2014) & $\begin{array}{l}\text { A duloxetina pode ser administrada com } \\
\text { segurança na vigência do aleitamento } \\
\text { materno. }\end{array}$ \\
\hline Chad et al. (2013) & $\begin{array}{l}\text { Irritabilidade, recusa alimentar e } \\
\text { problemas de sono são eventos } \\
\text { adversos relatados em bebês expostos a } \\
\text { antidepressivos. }\end{array}$ \\
\hline Sie et al. (2012) & $\begin{array}{l}\text { Os ISRSs* associam-se a efeitos } \\
\text { colaterais brandos e autolimitados. }\end{array}$ \\
\hline Chad et al. (2013) & $\begin{array}{l}\text { Eventos adversos são frequentes após } \\
\text { exposição à fluoxetina e ao citalopram. }\end{array}$ \\
\hline Di Scalea; Wisner (2009) & $\begin{array}{l}\text { A doxepina é associada à depressão } \\
\text { respiratória, sedação, hipotonia e vômitos. }\end{array}$ \\
\hline Sie et al. (2012) & $\begin{array}{l}\text { O uso de ISRSs* no período puerperal } \\
\text { parece aumentar o risco de anormalidades } \\
\text { comportamentais em longo prazo. }\end{array}$ \\
\hline
\end{tabular}

*ISRSs = Inibidores seletivos da recaptação de seratonina

Figura 3 - Estudos que apresentam os eventos adversos e/ou segurança da terapia antidepressiva no aleitamento materno

Por fim, ressalta-se que não há consenso sobre a superioridade da terapia farmacológica em detrimento às psicoterapias. A Figura 4 apresenta resultados de estudos que comparam essas duas modalidades terapêuticas no manejo da depressão pós-parto. 


\begin{tabular}{|c|c|}
\hline AUTORES/ANO & RESULTADOS \\
\hline Sharp et al. (2010) & $\begin{array}{l}\text { Fármacos tiveram eficácia clínica } \\
\text { significativamente superior que cuidados gerais } \\
\text { de suporte. }\end{array}$ \\
\hline Milgrom, et al. (2015) & $\begin{array}{l}\text { A terapia cognitivo comportamental foi superior } \\
\text { à sertralina e à terapia combinada após } 12 \\
\text { semanas de tratamento. }\end{array}$ \\
\hline $\begin{array}{l}\text { Di Scalea; Wisner } \\
\text { (2009) }\end{array}$ & $\begin{array}{l}\text { Fluoxetina e paroxetina, em monoterapia, } \\
\text { são igualmente eficazes à terapia cognitivo } \\
\text { comportamental. }\end{array}$ \\
\hline Bloch et al. (2012) & $\begin{array}{l}\text { As taxas de resposta terapêutica e } \\
\text { remissão da doença não são significati- } \\
\text { vamente alteradas ao adicionar sertralina } \\
\text { à psicoterapia. }\end{array}$ \\
\hline
\end{tabular}

Figura 4 - Resultados de estudos que compararam psicoterapia e psicofarmacologia no manejo da depressão pós-parto

\section{Discussão}

\section{Considerações para utilização de antidepressivos}

Os níveis de estrogênio em mulheres na vida reprodutiva são especialmente associados a interferências no mecanismo de ação de vários antidepressivos $^{(3)}$. Por isso, a psicofarmacoterapia para depressão pós-parto deve ser iniciada em monoterapia e na menor quantidade possível, com subsequente ajuste de doses $^{(8,17)}$. Antes de iniciar essa terapia antidepressiva, deve-se considerar que as puérperas podem ser excessivamente sensíveis a medicamentos devido aos efeitos hormonais nas enzimas hepáticas, alterações no volume de distribuição e maior concentração de proteínas que se ligam aos fármacos(8).

A resposta terapêutica com o uso de antidepressivos também é marcadas por variabilidades que podem ser explicadas por individualidades genéticas ${ }^{(3)}$. Assim sendo, a escolha do antidepressivo deveria, idealmente, considerar os genótipos da mãe e do bebê para melhores resultados. Após utilizar a farmacoterapia, as pacientes devem ser reavaliadas em até duas semanas e o tratamento deve se estender por um período mínimo de seis meses ${ }^{(8)}$.

Droga de escolha e introdução da terapia farmacológica com base na gravidade da doença

Diversos autores concordam que a sertralina deve ser a droga de escolha para o tratamento da depressão pós-parto ${ }^{(1,6,9,18)}$. Ademais, a preferência pela sertralina é justificada por sua baixa concentração no leite materno e no soro das crianças $^{(1)}$.

A sertralina associa-se a resposta terapêutica eficaz em até $67 \%$ das mulheres tratadas para depressão pósparto(6). Nesse estudo, a recorrência da doença foi de apenas $7 \%$ para aquelas que utilizaram sertralina e de $50 \%$ para as tratadas com placebo. Outros efeitos obtidos com o uso da sertralina na depressão pós-parto são: taxa de resposta significativamente maior que o placebo ( $53 \%$ vs. $21 \%$ ) e melhora da gratificação do papel materno, sem efeitos na interação materno-infantil(9,18).

Apesar de alguns autores ${ }^{(8)}$ defenderem a utilização de inibidores seletivos da recaptação de seratonina (ISRSs) no tratamento inicial da depressão puerperal leve a moderada pesquisadores(10) encontraram que não há benefício significativo em adicionar sertralina à psicoterapia no tratamento da depressão pós-parto leve a moderada, posto que, as taxas de resposta terapêutica e remissão da doença não são significativamente alteradas.

Nessa perspectiva, a maioria das diretrizes recomenda a utilização de antidepressivos como terapia inicial apenas na depressão pós-parto grave. Portanto, na depressão leve a moderada, as psicoterapias são preconizadas como tratamento inicial( ${ }^{(1)}$.

Deve-se ressaltar que os antidepressivos tricíclicos podem ser considerados o tratamento de primeira escolha, caso haja história de tratamento prévio com sucesso e não existam contra-indicações para seu uso, como a probabilidade de suicídio(13).

Utilização profilática de antidepressivos em mulheres susceptíveis

Uma questão bastante controversa no manejo da depressão pós-parto é a utilização profilática de antidepressivos em mulheres susceptíveis(11-12). Apesar de muito utilizados na prevenção da depressão pós-parto, a eficácia desse medicamentos é contestável e pouco se sabe sobre os possíveis efeitos colaterais, particularmente no período da amamentação. Evidências que apoiam o uso desses fármacos são limitadas a estudos com amostras escassas devido à insuficiência de dados na literatura(11-12).

Os impactos da patologia incentivam o tratamento farmacológico preventivo para mulheres com história de depressão pós-parto. Assim sendo, essas pacientes são candidatas a iniciar a sertralina para evitar que ocorram novos episódios da doença(13).

A utilização desses fármacos para prevenir a depressão pós-parto deve considerar as preferências da mulher, a gravidade dos sintomas e a história de episódios anteriores de depressão(11). Ademais, deve-se considerar também, que a segurança dos antidepressivos durante a gravidez é controversa. O uso desses fármacos tem sido associado à adaptação neonatal deficiente, risco de malformações cardiovasculares, hipertensão pulmonar persistente do neonato, parto prematuro, baixo peso ao nascer e transtornos psiquiátricos ${ }^{(1)}$.

Alguns autores ${ }^{(14)}$ encontraram benefícios em utilizar a sertralina para prevenir a depressão pós-parto. Contudo, essa terapia associou-se a maior risco de efeitos colaterais maternos. Considerando a nortriptilina, o autor não demostrou benefício na prevenção de episódios depressivos. 
Portanto, se for indicado o uso profilático de antidepressivo, é recomendado optar por um ISRS, de preferência, a sertralina. Deve-se ressaltar que, se a paciente já foi tratada para depressão puerperal, é razoável escolher o antidepressivo para o qual ela obteve a melhor resposta(6).

Antidepressivos, aleitamento materno e efeitos adversos no lactente

O leite humano é a fonte primária ideal de nutrientes e defesas imunológicas para o recém-nascido, fornecendo diversos benefícios à díade mãe-bebê(13). A maioria das diretrizes recomenda o incentivo à amamentação, independente da medicação antidepressiva utilizada ${ }^{1}$. Todavia, se a condição clínica da paciente suscitar a utilização de altas doses de antidepressivos ou forem prescritos múltiplos medicamentos, é provável que a amamentação tenha que ser interrompida(17).

O aleitamento materno influencia na aceitação da farmacoterapia pela mulher, posto que, muitas mães evitam utilizar medicações devido a preocupações com os efeitos adversos nos lactentes ${ }^{(15)}$.

As experiências iniciais negativas com o aleitamento materno, estariam associadas à ocorrência de sintomas depressivos, inclusive com a depressão pós-parto ${ }^{(16)}$. Hormônios lactogênicos, ocitocina e prolactina têm efeitos antidepressivos e ansiolíticos, sendo assim, podem estar envolvidos neste processo.

A dose infantil relativa é um importante parâmetro que mensura a dose de fármaco oferecida ao lactente por meio do leite. Valores inferiores a $10 \%$ da dose ajustada ao peso materno são considerados seguros na amamentação(19). A paroxetina e a sertralina produzem doses entre $0,5 \%$ e $3 \%$, enquanto a fluoxetina, venlafaxina e citalopram produzem níveis mais próximos ou até acima do limite de $10 \%$. Por isso, a sertralina e a paroxetina são as drogas recomendadas após o parto(19).

Além da sertralina e paroxetina, nortriptilina e imipramina estão entre os quatro fármacos com mais evidências para uso durante a amamentação(15). As drogas em questão associam-se a níveis séricos indetectáveis no lactente e a nenhum relato de efeito adverso em curto prazo. Nesse cenário, a duloxetina é outra droga que pode ser administrada com segurança a uma mulher que está amamentando, posto que, a exposição do bebê à droga é inferior a $1 \%$ da dose ajustada ao peso materno(20).

É possível afirmar que todos os antidepressivos utilizados na depressão pós-parto são detectáveis no leite materno e podem culminar em distúrbios do sistema nervoso central, gastrointestinal e respiratório em lactentes amamentados por mulheres que utilizaram essas drogas ${ }^{(17)}$. Sintomas como irritabilidade, recusa alimentar e problemas de sono são eventos adversos relatados em bebês expostos a antidepressivos através do leite materno. Esses eventos são mais frequentes após exposição à fluoxetina e ao citalopram ${ }^{(19)}$.

Os inibidores seletivos da recaptação da serotonina (ISRSs) são drogas cada vez mais utilizadas durante a gravidez e no período pós-parto. Apesar de excretados no leite materno, os efeitos colaterais costumam ser brandos e autolimitados, mas, ainda sim, demandam observação cuidadosa por pelo menos 48 horas $^{(21)}$.

A sertralina, apesar de considerada um ISRSs segura durante a amamentação, associa-se a efeitos adversos para o recém-nascido que incluem irritabilidade e recusa alimentar. Deve-se ressaltar que, se a criança nasceu prematura ou com baixo peso, esses efeitos são mais evidentes ${ }^{(21-22) .}$

Em contrapartida, a doxepina é um exemplo de antidepressivo tricíclico contraindicado para tratamento da depressão pós-parto. A referida droga é associada à depressão respiratória, sedação, hipotonia e vômitos(13).

Apesar de existirem poucas evidências sobre efeitos adversos graves em bebês expostos a antidepressivos através do leite materno, o efeito em longo prazo no desenvolvimento neurológico não foi adequadamente estudado(19). Nessa perspectiva, ao avaliar os efeitos em longo prazo dessas drogas, parece não haver repercussão no desenvolvimento cognitivo, mas anormalidades comportamentais parecem ser mais comuns ${ }^{(21)}$.

\section{Tratamento farmacológico vs. psicológico}

Muito se discute sobre qual seria a estratégia terapêutica mais eficaz no manejo da depressão pós-parto. Assim sendo, a escolha entre psicoterapia, farmacoterapia ou a combinação de ambas não é consenso na literatura.

Um estudo, ao comparar a eficácia clínica da terapia antidepressiva com os cuidados gerais de suporte em mães com depressão pós-parto, constatou que os fármacos foram, significativamente, superiores(23). Em contrapartida, alguns autores ${ }^{(24)}$ encontraram que a monoterapia com terapia cognitivo comportamental (TCC) é superior à sertralina e à terapia combinada após 12 semanas de tratamento da depressão pós-parto. Por sua vez, a fluoxetina e a paroxetina, em monoterapia, são igualmente eficazes à TCC, sendo que, nenhuma vantagem adicional é obtida ao combinar as terapias ${ }^{(13)}$.

Ainda sobre a combinação de terapias, outros autores(10) encontraram que as taxas de resposta terapêutica e remissão da doença não são significativamente alteradas ao adicionar sertralina à psicoterapia no tratamento da depressão pós-parto. Em contrapartida, a combinação de psicoterapia e antidepressivos deve ser o tratamento de escolha para depressão moderada a grave( ${ }^{(8)}$.

Quanto às preferências das mães, um autor constatou que mulheres com episódios prévios de 
depressão preferem os antidepressivos em detrimento à psicoterapia(6). Por outro lado, as mulheres que amamentam tendem a escolher a psicoterapia.

Ademais, como já mencionado, a maioria das diretrizes recomenda a utilização de antidepressivos na depressão pós-parto grave, sendo as psicoterapias preconizadas para doença leve a moderada(1).

\section{Conclusão}

A depressão pós-parto grave deve ser tratada com antidepressivos e a sertralina deve ser a droga de escolha. Baixa concentração no leite materno, resposta terapêutica eficaz e baixa recorrência da doença são alguns dos fatores que justificam a preferência pela referida droga.

Ressalta-se que não há consenso sobre a superioridade da terapia farmacológica em detrimento às psicoterapias no manejo da depressão puerperal, bem como a associação entre essas terapias.

A amamentação deve ser recomendada independente da medicação antidepressiva utilizada. Há evidencias que fundamentam o uso de sertralina, paroxetina, duloxetina, nortriptilina e imipramina na vigência do aleitamento materno. Em contrapartida, alguns autores advogam contra o uso de fluoxetina, citalopram e doxepina devido aos potenciais efeitos colaterais destas medicações.

Quanto à utilização profilática de antidepressivos, tem-se escassez de dados na literatura. Assim sendo, não há consenso quanto à utilização universal de fármacos em mulheres susceptíveis à referida desordem psiquiátrica. Por fim, frente às controversas quanto ao uso profilático de antidepressivos, emerge a necessidade de novos estudos com amostras representativas que visem validar ou restringir essa aplicabilidade dos psicofármacos.

\section{Agradecimentos}

Agradecemos as Faculdades Santo Agostinho pelo suporte para desenvolvimento do trabalho.

\section{Referências}

1. Molenaar NM, Kamperman AM, Boyce P, Bergink. Guidelines on treatment of perinatal depression with antidepressants: An international review. Aust N Z J Psychiatry. 2018; 52(4): 320-7. doi: $10.1177 / 0004867418762057$.

2. Anokye R, Acheampong E, Budu-Ainooson A, Obeng EI, Akwasi AG. Prevalence of postpartum depression and interventions utilized for its management. Annals Genl Psychiatry. 2018; 17(18):1-8. doi: 10.1186/ s12991-018-0188-0.

3. Keers R, Aitchison KJ. Gender differences in antidepressant drug response. Int Rev Psychiatry. 2010; 22(5): 485-500. doi: 10.3109/09540261.2010.496448.
4. Munk-Olsen T, Gasse C, Laursen TM. Prevalence of antidepressant use and contacts with psychiatrists and psychologists in pregnant and postpartum women. Acta Psychiatr Scand. 2012; 125(4): 318-24. doi: 10.1111/j.1600-0447.2011.01784.x.

5. Stein A, Murphy S, Arteche A, Lehtonen A, Harvey A, Craske $M G$, et al. Effects of reboxetine and citalopram on appraisal of infant facial expressions and attentional biases. J Psychopharmacol. 2012; 26(5):670-6. doi: 10.1177/0269881111421970.

6. Kim DR, Epperson CN, Weiss AR, Qisner KL. Pharmacotherapy of postpartum depression: an update. Expert Opin Pharmacother. 2014; 15(9): 1223-34. doi: 10.1517/14656566.2014.911842.

7. Souza MT de, Silva MD da, Carvalho R de. Revisão integrativa: o que é e como fazer. Einstein. 2010; 8(1): 102-6. doi: 10.1590/s1679-45082010rw1134

8. Langan RC, Goodbred A. Identification and Management of Peripartum Depression. Am Fam Physician. 2016; 93(10): 852-8. Available from: <https://pdfs.semanticscholar. org/37ad/f2f70f657411e65874f5d19e628838414c63.pdf> 9. Logsdon MC, Wisner K, Hanusa BH. Does maternal role functioning improve with antidepressant treatment in women with postpartum depression? J Womens Health. 2009; 18(1): 85-90. doi: 10.1089/jwh.2007.0635.

10. Bloch M, Meiboom H, Lorberblatt M, Bluvstein I, Aharonov I, Schreiber $S$. The effect of sertraline add-on to brief dynamic psychotherapy for the treatment of postpartum depression: a randomized, double-blind, placebo-controlled study. J Clin Psychiatry. 2012; 73(2): 235-41. doi: 10.4088/JCP.11m07117.

11. Molyneaux E, Telesia LA, Henshaw C, Boath E, Bradley E, Howard LM. Antidepressants for preventing postnatal depression (Review). Cochrane Database Syst Rev. 2018; 4(4363): 1-46. doi: 10.1002/14651858.CD004363.

12. Sharma V. A closer look at the preventative effect of antidepressants in postpartum depression. Arch Womens Ment Health. 2017; 20(2): 357-60. doi: 10.1007/ s00737-016-0709-2.

13. Di Scalea TL, Wisner KL. Pharmacotherapy of postpartum depression. Expert Opin Pharmacother. 2009; 10(16): 2593-607. doi: 10.1517/14656560903277202. 14. O'Connor E, Senger CA, Henninger ML, Coppola E, Gaynes BN. Interventions to Prevent Perinatal Depression: A Systematic Evidence Review for the U.S. Preventive Services Task Force. Evidence Synthesis. 2019; 321(6): 588-601. doi:10.1001/jama.2018.20865.

15. Di Scalea LT, Wisner KL. Antidepressant medication use during breastfeeding. Clin Obstet Gynecol. 2009; 52(3): 483-97. doi: 10.1097/GRF.0b013e3181b52bd6. 16. Figueiredo B, Dia CC, Brandão S, Canário C, NunesCosta R. Breastfeeding and postpartum depression: state of the art review. J Pediatr. 2013; 89(4): 332-8. doi: 10.1016/j.jped.2012.12.002. 
17. Hübner-Liebermann $B$, Hausner $H$, Wittman $M$. Recognizing and treating peripartum depression. Dtsch Arztebl Int. 2012; 109(24): 419-24. doi: 10.3238/ arztebl.2012.0419.

18. Hantsoo L, Ward-O'Brien D, Czarkowski KA, Gueorguieva R, Price LH, Epperson CN. A randomized, placebo-controlled, double-blind trial of sertraline for postpartum depression. Psychopharmacology. 2014;231(5): 939-48. doi: 10.1007/s00213-013-3316-1. 19. Chad L, Pupco A, Bozzo P, Koren G. Update on antidepressant use during breastfeeding. Can Fam Physician. 2013; 59(6): 633-4. Available from: <https:// www.ncbi.nlm.nih.gov/pmc/articles/PMC3681447/>

20. Andrade $\mathrm{C}$. The safety of duloxetine during pregnancy and lactation. J Clin Psychiatry. 2014; 75(12): 1423-7. doi: $10.4088 /$ JCP. 14 f09631.

21. Sie SD, Wenni JM, van Driel JJ, te Winkel AG, Boer $\mathrm{K}$, Casteelen $\mathrm{G}$ et al. Maternal use of SSRIs, SNRIs and NaSSAs: practical recommendations during pregnancy and lactation. Arch Dis Child Fetal Neonatal Ed. 2012; 97(6): 472-6. doi: 10.1136/archdischild-2011-214239.

22. Cuomo A, Maina G, Neal SM, De Montis G, Rosso G, Scheggi S, et al. Using sertraline in postpartum and breastfeeding: balancing risks and benefits. Expert Opin Drug Saf. 2018; 17(7): 719-25. doi: 10.1080/14740338.2018.1491546.

23. Sharp DJ, Chew-Graham C, Tylee A, Lewis G, Howard $\mathrm{L}$, Anderson I et al. A pragmatic randomised controlled trial to compare antidepressants with a community-based psychosocial intervention for the treatment of women with postnatal depression: the RESPOND trial. Health Technol Assess. 2010; 14(43): 1-153. doi: 10.3310/hta14430.

24. Milgrom J, Gemmill AW, Ericksen J, Burrows G, Buist A, Reece J. Treatment of postnatal depression with cognitive behavioural therapy, sertraline and combination therapy: a randomised controlled trial. Aust N Z J Psychiatry. 2015; 49(3): 236-45. doi: 10.1177/0004867414565474.

\section{Contribuição dos autores}

Concepção e planejamento do estudo: Thiago Guimarães Da Silva, Ivan Gilson Silva Moura. Obtenção dos dados: Thiago Guimarães Da Silva, Ivan Gilson Silva Moura. Análise e interpretação dos dados: Thiago Guimarães Da Silva, Pedro Fonseca De Vasconcelos, Ivan Gilson Silva Moura. Redação do manuscrito: Thiago Guimarães Da Silva, Pedro Fonseca De Vasconcelos. Revisão crítica do manuscrito: Pedro Fonseca De Vasconcelos, Ivan Gilson Silva Moura. Formatação: Pedro Fonseca De Vasconcelos.

Todos os autores aprovaram a versão final do texto.

Conflito de interesse: os autores declararam que não há conflito de interesse. 